\title{
ENSINO DE PROGRAMAÇÃO E APRENDIZADO DE MÁQUINA PARA ALUNOS DO ENSINO FUNDAMENTAL
}

Túlio R. Torres - tuliotorres7@hotmail.com

Matheus M. G. Costa-mmullergolz@gmail.com

Jorge L. Silva-jl.s.uca77@hotmail.com

Erivelton G. Nepomuceno-neponuceno@ufsj.edu.br

Marcos S.deOliveira-mso@ufsj.edu.br

Universidade Federal de São João del-Rei

Praça Frei Orlando, 170, Centro

36307-352 - São João del-Rei - Minas Gerais

André L.C.Ottoni-andre.ottoni@ufrb.edu.br

Universidade Federal do Recôncavo da Bahia

Rua Rui Barbosa, 710, Centro

44380-000 - Cruz das Almas - Bahia

Bruno de P.O. Paiva - bruno.paiva@educacao.mg.gov.br

E. E. Tomé Portes Del Rei

Rua Cel. José de Assis Sobrinho, 49, Matozinhos

36305-162 - São João del-Rei - Minas Gerais

Resumo: Este artigo descreve a metodologia e as atividades de ensino e extensão realizadas com alunos do ensino fundamental da Escola Estadual Tomé Portes Del Rei. Neste trabalho serão apresentadas ferramentas, plataformas, linguagens de programação, estratégias de ensino e resultados obtidos no ensino de programação e inteligência artificial para alunos de ensino fundamental do $6^{\circ}$ ao $9^{\circ}$ ano. $O$ objetivo principal foi gerar interesse e motivação de alunos pela área de programação e inteligência artificial partindo de um ponto sem nenhuma experiência por parte dos participantes no tema e com foco em proporcionar o contato a estes assuntos ao público mais jovem. Procurou-se despertar o desejo de aprendizado sobre tais temas a fim de inspirá-los nas carreiras de ciências exatas além de usar tais conhecimentos em suas futuras profissões.

Palavras-chave: Aprendizado de máquina. Ensino a distância. Programação. 


\section{INTRODUÇÃO}

O “Aprendizado por Reforço: Engenharia e Estatística Impulsionando a Sociedade” é o nome do primeiro projeto indissociável da Universidade Federal de São João del-Rei (UFSJ). Este projeto reúne pesquisa, ensino e extensão em torno de dois temas muito recorrentes atualmente, programação e inteligência artificial. Este artigo descreve a metodologia de atividades de ensino e extensão realizada com os alunos do ensino fundamental da Escola Estadual Tomé Portes Del Rei. Nesse trabalho serão apresentadas algumas ferramentas (CS First, Arduino, Scratch e VisualG), uma plataforma (Google Meet), linguagens de programação e similares (C e Portugol), estratégias de ensino (Ensino a distância - EAD) e resultados obtidos no ensino de programação e inteligência artificial.

Diversos especialistas defendem a importância do ensino de programação desde a infância (GERALDES, 2014). Mitchel Resnick, criador do programa Scratch que será utilizado como forma de ensino neste trabalho, define que "habilidades de lógica e de programação serão úteis não apenas para cientistas, mas para qualquer pessoa, independentemente da idade, da experiência, do interesse ou da profissão que optar por seguir" (SERRANO, 2014). Resnick também diz que propostas como o Scratch levam o estudante a um processo de aprendizado contínuo, num ciclo que envolve criar, experimentar, compartilhar e refletir, resultando em expansão de suas habilidades, conhecimentos e competências (RESNICK, 2009).

Espera-se com este trabalho fomentar o interesse pela programação e despertar nos alunos do ensino fundamental o desejo de aprendê-la, desde os anos iniciais. E com isto, possivelmente inspirá-los a utilizá-la em suas futuras profissões, na engenharia, matemática ou ciências como um todo.

Este artigo está dividido nas seguintes seções: fundamentação teórica (em que serão apresentadas algumas ferramentas e conceitos utilizados), metodologia (onde será descrito o processo de desenvolvimento de material didático, a seleção dos alunos, e como ocorreram as aulas de programação remota, além do desenvolvimento de aulas EAD de aprendizado de máquina) e, por fim, serão apresentados os resultados obtidos, a avaliação dos alunos e as conclusões.

\section{FUNDAMENTAÇÃO TEÓRICA}

\subsection{Scratch}

O Scratch é uma linguagem de programação voltada para usuários sem experiência em programar. O software foi criado por pesquisadores da Lifelong kindergarten do Laboratório de Mídias do Massachusetts Institute of Technology (MIT) e tem como público alvo crianças a partir de oito anos e adolescentes (DIAS ET AL., 2014).

O programa oferece aos usuários uma interface visual para programação. Isso facilita a aprendizagem de alguns conceitos como entrada e saída, tipos de dados, variáveis, estruturas de controle, operadores e arrays. Além disso o Scratch possui uma interface onde os comandos são montados por meio de blocos virtuais. Assim o usuário consegue construir histórias, jogos entre outras coisas (DIAS ET AL., 2014).

A Figura 1 apresenta a interface do programa Scratch. Além disso, na Figura 1 é apresentado um exemplo de implementação (jogo Pong) através de blocos e conceitos de lógica de programação. 
Figura 1: Jogo Pong criado no Scratch.

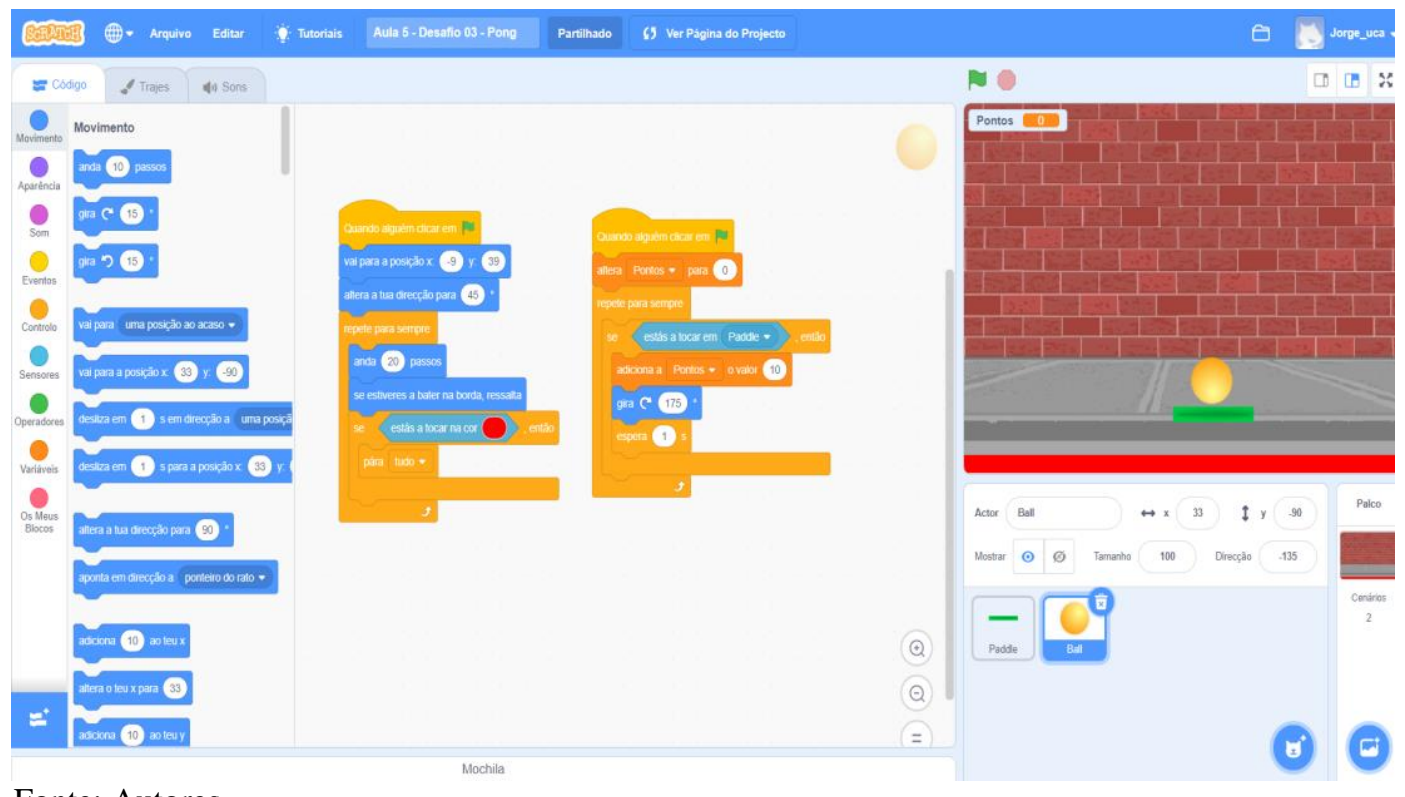

Fonte: Autores.

\subsection{VisualG}

O VisualG foi o programa utilizado para apresentar aos alunos uma abordagem prática de programação em códigos. Ele é "um programa que permite criar, editar, interpretar e que também executa os algoritmos em português estruturado (Portugol) como se fosse um programa de computador" (VisualG, 2017). O Portugol é uma nomenclatura usual para linguagem de programação baseada em pseudocódigo, utilizada para apresentar uma abordagem prática aos alunos.

O VisualG representa um Ambiente de Desenvolvimento Integrado, do inglês Integrated Development Environment - IDE, onde o aluno pode escrever seus pseudocódigos e observar seus resultados. O VisualG permite que os alunos de países de língua portuguesa possam ter uma iniciação com o mundo de linguagens de programação mais simplificada (DE SOUZA, 2009). Um ótimo exemplo a ser usado são os comandos de repetição e de saída de dados. Em linguagens mais estabelecidas o comando de repetição pode ser "while", em Portugol você pode utilizá-lo com a palavra "enquanto". Em outras linguagens é comum que o comando de saída de dados seja o "print", já em Portugol é utilizado o "escreva". Assim possibilitando e facilitando para o aluno o entendimento do que aquele código executa, como mostra a Figura 2.

Utilizando-se de uma interface amigável, como pode-se observar na Figura 2, separando o console, área de variáveis, seções de declaração de variáveis, entre outras, o uso desta ferramenta se mostra bastante produtivo, por permitir que desde o início os estudantes tenham contato com um ambiente de desenvolvimento próximo do que encontrarão na vida real. (DE SOUZA, 2009). 
Figura 2: Código desenvolvido no VisualG 3.0

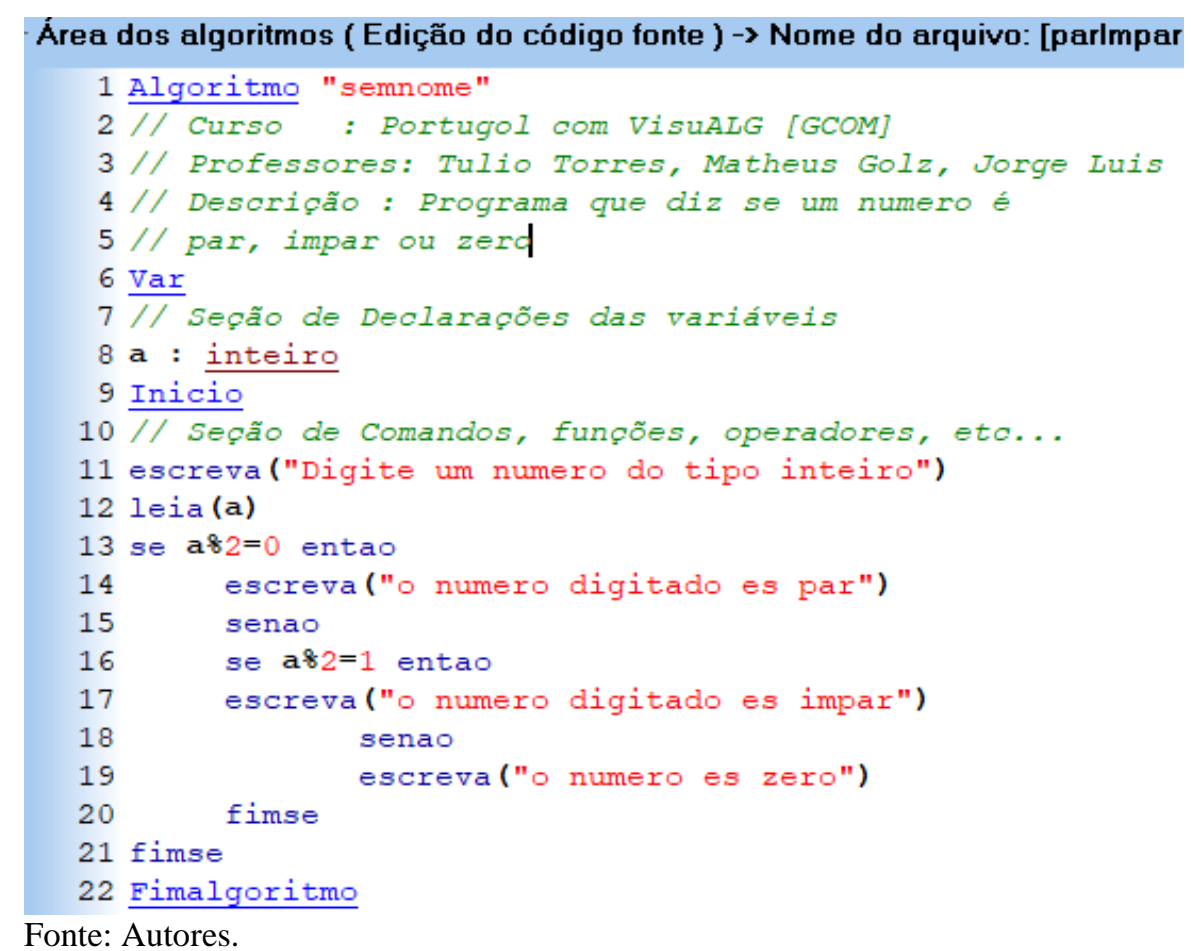

\subsection{Aprendizado por Reforço}

A base para o aprendizado por reforço parte do princípio de exploração autônoma por parte do algoritmo. De início são definidos o agente, aquele que irá executar as ações, o ambiente, onde as ações serão executadas, o estado, que indica ao agente qual a situação do ambiente e as recompensas, cuja função é servir como feedback ao agente da qualidade de sua tomada de decisão (SUTTON; BARTO, 2018). A partir desse ponto é utilizado a teoria de Processos de Decisão de Markov. Os Processos de Decisão de Markov são uma formalização do processo de tomada de decisões sequenciais na qual é levado em consideração como as ações influenciam as recompensas imediatas bem como as subsequentes.

Através da interação com o ambiente o agente identifica quais ações geram melhores recompensas de forma que consiga avaliar quais as melhores ações para cumprir seu objetivo, o de maximizar sua pontuação.

Os dois algoritmos clássicos do aprendizado por reforço são o Q-Learning e SARSA. O primeiro tem como princípio de funcionamento a atualização dos valores dos estados com base na maior recompensa possível de ser obtida no estado posterior, enquanto o segundo analisa os valores de estado de acordo com a política sendo utilizada. Utilizando-se dos conceitos de aprendizado por reforço pode-se abordar uma gama de problemas. A seguir tem-se exemplos que foram abordados nos vídeos produzidos: 1. Navegação autônoma (OTTONI, 2016), consiste na resolução de problemas de deslocamento dentro de um ambiente conhecido ou não; 2. Caixeiro Viajante (OTTONI, 2015), um algoritmo que tem como objetivo a otimização de rotas com base em pontos no qual o agente deve passar; 3. Mochila Multidimensional (OTTONI, 2017), um problema de otimização combinatória cujo objetivo é encontrar uma combinação de itens que maximize a quantidade de carga que pode ser transportada. 


\section{METODOLOGIA}

\subsection{Desenvolvimento do material didático}

As aulas de programação foram construídas utilizando uma base de apoio de um material pré-existente do Google. O material faz parte do curso CS First e faz uso conjunto da Ferramenta Scratch, sendo recomendado para estudantes dentro da faixa etária de 9 a 14 anos, em coerência com a faixa etária dos estudantes que participaram das atividades aqui descritas.

O material é de acesso livre e pode ser utilizado para ensino assim como foi feito para este trabalho, na qual a ementa e a identidade visual para as apresentações foram mantidas semelhantes.

O Scratch foi utilizado para dar as noções iniciais de lógica, execução sequencial, e a dos blocos de utilização comuns nas diferentes linguagens de programação, como estruturas condicionais e repetições. Após o Scratch, foi utilizado o Portugol para aproximar um pouco mais das linguagens de programação tradicionais.

As aulas foram especificadas com duração curta, de no máximo 40 minutos, visto o risco de desistência por parte dos alunos e considerando a não existência do caráter obrigatório das aulas. Durante o correr do curso também procurou-se estimular a curiosidade dos participantes com aulas que partiam dos seus interesses e os mesclavam com a ementa do curso. Como exemplo, foi feita uma união do desejo de conhecer o desenvolvimento de jogos com a apresentação de um software que funciona como motor gráfico e é utilizado para representações de projetos arquitetônicos (fotorrealismo) além do desenvolvimento de jogos. Para ampliar o interesse e o conhecimento dos estudantes por robótica e programação, os mesmos também foram apresentados a plataforma Arduino que funciona como uma boa porta de entrada para aprendizado de programação e eletrônica básica, visto sua linguagem de alto nível e popularidade da plataforma tendo referências online para diversos projetos já bem detalhados.

Todos os conteúdos produzidos incluindo aulas e vídeos do YouTube podem ser encontrados na página do projeto, disponível em: www.ufsj.edu.br/ar/.

\subsection{Seleção dos alunos}

Inicialmente foi feita uma seleção a partir de questões que envolviam lógica de programação, porém no dia a dia dos alunos. Um exemplo foi uma atividade que consistia em que os alunos tentassem transcrever o passo a passo e de maneira ordenada, quais ações eles executavam para escovar os dentes todos os dias. Além dessa, questões sobre familiaridade com o computador e interesse por tecnologias também foram abordadas.

Porém com a ocorrência da pandemia de Covid-19, foi necessário descartar a seleção anterior. Foi então possibilitado que os alunos pudessem acompanhar as aulas de forma online. Com intermédio do professor Bruno de Paula, foi mantido o contato com os alunos interessados e criado um grupo virtual no whatsapp, onde este se tornou o principal meio de comunicação entre os alunos do ensino fundamental e os 3 alunos de graduação que ministraram as aulas de forma $\mathrm{EAD}$.

\subsection{Aulas de Ensino Remoto de Programação de Computadores}

Um dos principais objetivos deste projeto consistia nas aulas presenciais para os alunos da Escola Estadual Tomé Portes Del Rei. Infelizmente estas foram canceladas devido a pandemia. Porém o cronograma foi reajustado para que ocorressem de maneira online. Como plataforma para que as aulas fossem aplicadas foi utilizado o Google Meet. 
A introdução do curso foi apresentada usando a ferramenta Scratch, contemplando conceitos como programação sequencial, estruturas simples, repetições, condicionais e operadores.

A última etapa realizada nas Aulas de Ensino Remoto, foi a utilização do VisualG, como ferramenta de compilação para os códigos (pseudocódigos) em Portugol. Foram ensinados os comandos e regras de sintaxe básicas para a compilação. Nesta etapa foi possível apresentar aos alunos como são feitas as mudanças de estados, a criação de variáveis, uso de operadores lógicos e aritméticos, estruturas de repetição e condicionais, tudo isso em linhas de código, o que se assemelha mais da programação "real". A seguir está descrito todo o conteúdo ministrado bem como as divisões dos conteúdos para cada aula.

Aula 01: Apresentação do projeto e Scratch, o que é programação.

Aula 02: História do computador e lógica.

Aula 03: Estudo da estrutura de repetição.

Aula 04: Exercícios das Aulas 01, 02 e 03.

Aula 05: Estudo das estruturas condicionais.

Aula 06: Resolução do desafio do labirinto.

Aula 07: Operações matemáticas no Scratch.

Aula 08: Correção de exercício, apresentação da plataforma Arduino e sua programação.

Aula 09: Desenvolvimento do jogo Pac-Man.

Aula 10: Desenvolvimento de um jogo utilizando o Mouse.

Aula 11: Programação de um semáforo em Arduino.

Aula 12: Apresentação do VisualG e comandos de programação em código.

Aula 13: Comandos no VisualG: variáveis, operadores, fluxo de dados, repetições e condicionais.

Aula 14: Operações aritméticas no VisualG.

Aula 15: Robótica: Aplicações e Exemplos.

Aula 16: Jogos, Arquitetura Virtual e Motores Gráficos.

Aula 17: Resolução de atividades utilizando estruturas condicionais e de repetição no VisualG.

Aula 18: Resolução de atividades utilizando estruturas condicionais e de repetição no VisualG.

Muitos dos tópicos abordados foram desenvolvidos com exemplos cotidianos, seja através das explicações sobre aplicação de lógica por trás do funcionamento de eletrodomésticos e carros, seja através da elaboração de aulas sobre os tópicos de interesse dos alunos desenvolvendo-os com os conceitos ensinados.

\subsection{Aulas de EAD sobre Aprendizado de Máquina: Vídeoaulas}

As aulas gravadas têm o propósito de trazer o contexto de aprendizado de máquina, inteligência artificial e aprendizado por reforço para os alunos. Utilizando-se um vocabulário de fácil compreensão para os iniciantes no contexto de programação e inteligência artificial, são abordados os conceitos iniciais deste campo, as formas de funcionamento do aprendizado por reforço, tanto de forma mais geral quanto seus dois algoritmos mais clássicos Q-Learning e SARSA e casos de aplicação.

A plataforma escolhida para a publicação das aulas foi o YouTube, visto sua popularidade dentre o público alvo de adolescentes e jovens, favorecendo maior acessibilidade e visualização do conteúdo.

Os conteúdos são transmitidos através de apresentações com slides ou gravações de telas nos casos dos programas sobre a qual são editadas as gravações de voz correspondentes. 
Algumas das aulas possuem atividades propostas para aos que assistem como forma de tornar a experiência mais dinâmica e auxiliar o aprendizado.

A sequência de aulas foi pensada de forma a seguir um aumento gradual no nível de complexidade, onde os conteúdos de uma aula são construídos aproveitando-se os assuntos já abordados.

\section{Conteúdo programático do curso "Introdução ao Aprendizado por Reforço":}

Aula 1: O que é inteligência artificial, aprendizado de máquina, referências, aplicações e subdivisões da área.

Aula 2: Conceito de inteligência, racionalidade, medidas de desempenho, autonomia, tipos de agente e ambiente.

Aula 3: O aprendizado por reforço: conceitos e aplicações.

Aula 4: Aprendizado por diferença temporal, modelos matemáticos SARSA e Q-Learning, discussão dos modelos e parâmetros.

Aula 5: Aplicação do aprendizado de máquina em problema de navegação autônoma, algoritmos, definições e demonstrações em exemplo.

Aula 6: Aplicação do aprendizado por reforço na resolução do problema do Caixeiro Viajante. Aula 7: Aplicação do aprendizado por reforço na resolução do problema da Mochila Multidimensional.

\section{RESULTADOS}

\subsection{Análise inicial}

Uma análise inicial dos resultados procurou identificar o interesse dos alunos pela participação das aulas a distância e o número efetivo de participantes. A princípio, 26 alunos manifestaram interesse em participar das aulas em um levantamento anterior a pandemia, com isso havia sido necessário aumentar o número de turmas para comportar todos os alunos. Após a modificação das atividades de forma a dar continuidade durante o período de isolamento social foi refeita a seleção, resultando em 8 participantes. A redução expressiva indica a maior dificuldade de se atender certos requisitos necessários para o cumprimento das atividades, como conexão à internet para o acompanhamento das aulas e computadores para a realização das tarefas.

A princípio o formato proposto das aulas era composto pela apresentação do conteúdo e discussão das atividades propostas da aula anterior. Estas atividades eram propostas para serem realizadas pelos estudantes individualmente e enviadas para correção. Devido a um baixo rendimento caracterizado pelo baixo número de atividades enviadas foi feita uma alteração do formato das aulas. Nesse novo formato todas as atividades eram realizadas durante o tempo da aula, não sendo mais necessário seu envio. Com isso foi possível que os alunos tivessem o contato com os problemas e suas resoluções bem como as discussões que surgiram ao longo de seu desenvolvimento.

\subsection{Resultado dos questionários}

Para realizar a análise dos resultados obtidos com as aulas foram criados formulários de preenchimento online. Esses formulários consistem de 5 questões referentes aos conteúdos abordados na sua respectiva aula. Os indicadores a seguir revelam os resultados obtidos com cada um dos questionários. 
Tabela 1 - Indicadores obtidos pelos questionários.

\begin{tabular}{c|c|c|}
\hline Número do Questionário & Número de respostas & Percentual médio de acertos \\
\hline 1 & 7 & $69,57 \%$ \\
\hline 2 & 3 & $86,67 \%$ \\
\hline 3 & 2 & $50,00 \%$ \\
\hline
\end{tabular}

Fonte: Autores.

Número total de respostas: 12

Média geral de acertos: $68,41 \%$

Os questionários aplicados tiveram como objetivo verificar se os alunos conseguiram assimilar e entender os conceitos de Aprendizado de Máquina apresentados nos vídeos. A partir de uma análise das respostas identificou-se que os erros dos alunos foram causados principalmente pela falta de familiaridade com as nomenclaturas de Inteligência Artificial e conceitos básicos de Aprendizado por Reforço. Como exemplo pode-se citar um caso onde os alunos descreveram programação dinâmica ao tentar se referir ao treinamento de modelos de aprendizado supervisionado, tópicos que de certa forma estão relacionados e podem ser confundidos.

\subsection{Depoimento dos bolsistas}

A execução deste trabalho também pode ser avaliada pela perspectiva dos alunos bolsistas a qual tiveram a experiência de ministrar as aulas. De forma geral, o contato pode ser descrito como "enriquecedor", ao dar a oportunidade para que estudantes se coloquem no papel de professores. A experiência foi de grande valia tanto para uma maior consciência da importância do processo educativo como um todo, suas dificuldades e gratificações bem como para as futuras escolhas profissionais de cada um dos bolsistas.

\section{CONCLUSÃO}

O objetivo deste trabalho se deu no ensino de programação e inteligência artificial para alunos do ensino fundamental de uma escola pública da cidade de São João del-Rei. O intuito foi demonstrar aos alunos um novo mundo em que estarão cada vez mais imersos e poder incentivá-los a seguir suas vidas futuras nesta área. A análise dos resultados mostra que para o grau de rigor do conhecimento com que foram tratados os assuntos, que por sua vez tem um requisito de conhecimento prévio bastante extensivo, foram obtidos resultados satisfatórios. Ao considerar a análise do ponto de vista que os formulários, devido a sua característica de respostas fechadas, mascaram a interpretação dos resultados aos dois extremos do espectro, o completo entendimento de um tópico ou o oposto, podemos concluir que mesmo os alunos que não obtiveram uma boa classificação em termos numéricos ainda obtiveram um bom aprendizado dos conceitos como foi discutido anteriormente.

A boa interação com os alunos e as discussões resultantes da apresentação de conceitos antes desconhecidos a eles em aplicações onde eles tinham interesse também possibilitou uma nova perspectiva para que eles pudessem compreender as máquinas, computadores e jogos preferidos sobre um novo olhar. Tal resultado acaba por estimular os alunos a crescer seu interesse nas áreas de ciências e tecnologia, um bom desfecho para o projeto. Também foi de grande valia a experiência de ensino para os bolsistas do projeto, muitos dos quais tiveram seu primeiro contato com docência, mesmo tendo ocorrido em um formato não convencional. 
Apesar de todas as dificuldades impostas pela pandemia, foi possível a realização de aulas virtuais e a criação de um material de ensino sobre aprendizado de máquina. Porém dentre todos os objetivos conquistados o mais importante foi poder despertar nos alunos o interesse por programação e inteligência artificial, como pode-se observar nas reações positivas com as aulas e no descobrimento destas áreas e sua influência em outros temas de interesse dos estudantes. Espera-se que os alunos persistam nos estudos de programação e que nos próximos anos alguns deles estejam cursando uma graduação e estudando mais a fundo sobre linguagens e lógicas de programação. Além de utilizando os conceitos aprendidos, tanto academicamente, profissionalmente ou até mesmo nas suas vidas pessoais.

Como continuidade a este trabalho os alunos serão direcionados a outros projetos de ensino presentes na UFSJ, com objetivo de explorar e aprofundar os conhecimentos apresentados nos cursos.

Em trabalhos futuros, espera-se poder aprofundar os conceitos de programação lecionando matérias presentes em grades curriculares de ensinos técnicos e acadêmicos. Como, por exemplo, ensinar linguagens de programações efetivas no mercado de trabalho e na academia. Espera-se também, realizar um evento/gincana em que se possa colocar em prática as habilidades adquiridas no decorrer do projeto.

\section{Agradecimentos}

Agradecemos à CAPES, CNPq/INERGE, FAPEMIG, UFRB e UFSJ (Edital $\mathrm{n}^{\circ}$ 001/2019/Reitoria).

\section{REFERÊNCIAS}

DE SOUZA, Cláudio Morgado. VisuAlg-Ferramenta de apoio ao ensino de programação. Revista Eletrônica TECCEN, v. 2, n. 2, p. 01-09, 2009.

DIAS, Klissiomara; SERRÃO, Miquéia. A linguagem Scratch no ensino de programação: Um relato de experiência com alunos iniciantes do curso de licenciatura em computação. In: Anais do XXII Workshop sobre Educação em Computação. SBC, 2014. p. 189-198.

GERALDES, W. B. Programar é bom para as crianças? Uma visão crítica sobre o ensino de programação nas escolas. Texto Livre: Linguagem e Tecnologia, v. 7, n. 2, p. 105-117, 2014.

OTTONI, André. L. C.; NEPOMUCENO, Erivelton. G.; CORDEIRO, Lara. T.; LAMPERTI, Rubisson. D.; OLIVEIRA, Marcos. S. Análise do Desempenho do Aprendizado por Reforço na Solução do Problema do Caixeiro Viajante. SBAI 2015 - Simpósio Brasileiro de Automação Inteligente, 2015, Natal - RN.

OTTONI, A. L. C.; NEPOMUCENO, E. G.; OLIVEIRA, M. S.; CORDEIRO, L. T.; LAMPERTI, R. D. . Análise da influência da taxa de aprendizado e do fator de desconto sobre o desempenho dos algoritmos Q-learning e SARSA: aplicação do aprendizado por reforço na navegação autônoma. Revista Brasileira de Computação Aplicada, v. 8, p. 44-59, 2016.

OTTONI, A. L. C.; NEPOMUCENO, E. G. ; OLIVEIRA, M. S. Análise do desempenho do aprendizado por reforço na solução do problema da mochila multidimensional. REVISTA BRASILEIRA DE COMPUTAÇÃO APLICADA, v. 9, p. 56-70, 2017. 
RESNICK, Mitchel et al. Scratch: programming for all. Communications of the ACM, v. 52, n. 11, p. 60-67, 2009.

SERRANO, F. Geração Geek. Revista Exame Informática. São Paulo: Editora Abril, 2014.

SUTTON, Richard S.; BARTO, Andrew G. Reinforcement learning: An introduction. MIT press, 2018.

VisualG. Disponível em: https://visualg3.com.br. Acesso em: 28 jul. 2020.

\title{
TEACHING PROGRAMMING AND MACHINE LEARNING TO MIDDLE SCHOOL STUDENTS
}

\begin{abstract}
This article describes the methodology and teaching and extension activities carried out with elementary school students at the Tomé Portes Del Rei State School. In this work, tools, platforms, programming languages, teaching strategies and results obtained in the teaching of programming and artificial intelligence for elementary school students from 6th to 9th grade. The main objective was to generate interest and motivation of students in the area of programming and artificial intelligence, starting from a point with no experience on the part of the participants in the theme and with a focus on providing contact with these subjects to the younger audience. We sought to awaken the desire to learn about such topics in order to inspire them in the careers of exact sciences in addition to using such knowledge in their future professions.
\end{abstract}

Keywords: Machine learning. E-learning. Programming. 